\title{
On the Stability of Robot Kinesthetic Guidance in the Presence of Active Constraints
}

\author{
Theodora Kastritsi ${ }^{1,2}$, Dimitrios Papageorgiou ${ }^{1,2}$ and Zoe Doulgeri ${ }^{1,2}$
}

\begin{abstract}
The stability of a robot subject to active constraints under the exertion of a human force is analyzed. Artificial potentials that are used to create a barrier on constraint surfaces are revisited and the robot's stability in the presence of active constraints is examined in terms of output passivity and state boundedness. It is shown that under input forces of finite energy the state is bounded and hence the motion is confined within the constraint region. However, the quality of response depends on the type of input and may include undesirable transients for physical human robot interaction (pHRI) applications.
\end{abstract}

\section{INTRODUCTION}

In intentional pHRI tasks like kinesthetic guidance, an important key issue that needs to be addressed by the robot's controller concerns the robot's quality-of-performance. To this aim the notion of active constraints or virtual fixtures was introduced that enhances pHRI performance in terms of precision, cognitive load and user effort. Active constraints were firstly introduced in tele-robotic manipulation [1], [2], [3] and have been utilized in surgical [4], [5], [6], [7], industrial [8], [9], or even in underwater robotic tasks [10]. They encourage motion inside a constraint region by acting repulsively away from forbidden regions thus avoiding obstacles in set point robot control problems [11] or assisting the user in pHRI tasks. They have also been used in region reaching control methods which is a generalization of the set point control problem; region reaching is needed in visual servoing to ensure that all features are within the field of view during motion [12],[13]. Active constraints are mostly imposed via artificial potential functions that repel the motion away from the constraints. Regarding the system's stability characteristics, most of the robotic's literature only partially analyze it. In pHRI related papers the analysis is confined to the proof of the system's passivity with respect to the system velocity output. In the set point and region reaching control problem, the stability analysis is confined to the goal's global asymptotic stability without considering the effect of external inputs that may arise from noisy state measurements, unintentional contacts and/or human kinesthetic guidance forces. The latter appears in cases of a robot under impedance control without inertia shaping with known task related equilibrium.

\footnotetext{
${ }^{1}$ Authors are with Aristotle University of Thessaloniki, Department of Electrical and Computer Engineering, Thessaloniki 54124, Greece. e-mail: tkastrit@ece.auth.gr,dimpapag@eng.auth.gr doulgeri@eng.auth.gr

${ }^{2}$ Authors are also Research Associate at Center for Research and Technology Hellas (CERTH), 57001 Thessaloniki, Greece. e-mail: theokast@iti.gr, dimpapag@iti.gr, doulgeri@iti.gr

This work is funded by the EU Horizon 2020 research and innovation programme under grant agreement No: 732515, project SMARTsurg.
}

In this work, we analyze the stability of a robot subject to active constraints under the exertion of an external input like a human guidance force. Issues of passivity, constraint satisfaction and state boundedness are examined and demonstrated via the simulation of a two dof revolute prismatic manipulator. The analysis makes use of the findings regarding the characterization of integral input to state stability reported in [14].

\section{A SURVEY ON THE ARTIFICIAL POTENTIALS FOR CONSTRAINT SATISFACTION}

Consider a point-mass robot moving within an mdimensional space, e.g., the end-effector of a manipulator moving in 3-dimensional task space, or the configuration of a manipulator in $\mathrm{C}$-space. Let the $\mathrm{m}$-dimensional space include a restricted region defined as a closed set $\mathcal{O} \subset \mathbb{R}^{\mathrm{m}}$, which should not be violated by the robot e.g., an obstacle. Let $\mathbf{p} \in \mathbb{R}^{\mathrm{m}}$ be the generalized position of the robot.

A common approach for constraint satisfaction or collision avoidance is the utilization of Artificial Potential (AP) fields that create a barrier on the constraint surfaces and become negligible beyond. APs are positive scalar functions of a distance metric $d(\mathbf{p}): \mathbb{R}^{\mathrm{m}} \mapsto \mathbb{R}_{\geq}$from the constraint boundary of $\mathcal{O}$, being zero on the boundary. The minimum Euclidean distance between the robot position $\mathbf{p}$ and the restricted region $\mathcal{O}$, is often utilized :

$$
d(\mathbf{p})=\min _{\mathbf{p}^{*} \in \mathcal{O}} \sqrt{\left(\mathbf{p}-\mathbf{p}^{*}\right)^{T}\left(\mathbf{p}-\mathbf{p}^{*}\right)}
$$

AP fields $V_{c}(d(\mathbf{p}))=V_{c}(\mathbf{p})$ are designed within the constraint-free space $\Omega=\mathbb{R}^{\mathrm{m}}-\mathcal{O}$, and usually fall within a category characterized by the following properties:

- $V_{c}(\mathbf{p})$ is a continuous differentiable function, $\forall \mathbf{p} \in \Omega$

- $V_{c}(\mathbf{p}) \geq 0, \forall \mathbf{p} \in \Omega$

- $V_{c}(d(\mathbf{p})) \rightarrow \infty$ if and only if $d(\mathbf{p}) \rightarrow 0$

- There exists a minimum-value set $\mathcal{S}=\{\mathbf{p} \in \Omega$ : $\left.\frac{\partial V_{c}(\mathbf{p})}{\partial \mathbf{p}}=\mathbf{0}\right\}$

To impose a constraint related artificial potential field, the following control signal is applied $\mathbf{u}_{c} \in \mathbb{R}^{\mathrm{m}}$ :

$$
\mathbf{u}_{c}=-\frac{\partial V_{c}(\mathbf{p})}{\partial \mathbf{p}} \text {. }
$$

In the case of robot kinesthetic guidance, the control signal usually contains a gravity compensation term $\mathbf{u}_{g}$ and an additive dissipative force term of the form

$$
\mathbf{u}_{d}=-\mathbf{D}_{p} \dot{\mathbf{p}}
$$


with $\mathbf{D}_{p} \in \mathbb{R}^{\mathrm{m} \times \mathrm{m}}$ being a positive constant matrix. In case the robot is desired to autonomously reach a position target, a potential that is attractive to the goal $\mathbf{p}_{T}$ is added to the system (e.g. $\left\|\mathbf{p}-\mathbf{p}_{T}\right\|^{2}$ ) and applied via an extra control signal. In this work we are interested in the stability analysis of the robot under an input guidance force exerted by the user in the presence of virtual constraints. Before motivating our work we briefly present the APs and respective control signals that have been proposed in the literature for constraint satisfaction.

One of the first works based on this approach was introduced by Khatib et al. [11], [15], In [15], the following AP function is proposed:

$$
V_{c}(\mathbf{p})= \begin{cases}\frac{\mathrm{k}}{2}\left(\frac{1}{d(\mathbf{p})}-\frac{1}{\mathrm{~d}_{0}}\right)^{2} & \text { if } d(\mathbf{p}) \leq \mathrm{d}_{0} \\ 0 & \text { otherwise }\end{cases}
$$

where $\mathrm{k}>0$ is a constant parameter and $\mathrm{d}_{0} \in \mathbb{R}_{>}$is the distance determining the area-of-influence of the potential field. Let us denote by $\mathcal{A}$ this area of influence, $\mathcal{A}=\{\mathbf{p} \in$ $\left.\Omega: \frac{\partial V_{c}(\mathbf{p})}{\partial \mathbf{p}} \neq 0\right\}$. Hence, the minimum-value set $\mathcal{S}=\Omega-$ $\mathcal{A}$. We get the control signal (2) by differentiating (4) with respect to $\mathbf{p}$ :

$\frac{\partial V_{c}(\mathbf{p})}{\partial \mathbf{p}}=- \begin{cases}\mathrm{k}\left(\frac{1}{d(\mathbf{p})}-\frac{1}{\mathrm{~d}_{0}}\right) \frac{1}{d(\mathbf{p})^{2}} \frac{\partial d(\mathbf{p})}{\partial \mathbf{p}} & \text { if } d(\mathbf{p}) \leq \mathrm{d}_{0} \\ 0 & \text { otherwise }\end{cases}$

Multiple restricted regions $\mathcal{O}_{i} \subset \mathbb{R}^{\mathrm{m}}$, with $i=1, \ldots, \mathrm{n}$ can be easily accommodated by superimposing the AP fields and summing their respective gradients in the control. The constraint-free space is then given by $\Omega=\mathbb{R}^{\mathrm{m}}-\bigcup_{i}^{\mathrm{n}} \mathcal{O}_{i}$. An example utilizing the potential function (4) for two constraints in a 2-D world produced by adding the individual potentials is illustrated in Figure 1, for spherical obstacles (Figure 1.a and b) and planar constraints (Figure 1.c and d)

Remark 1. A simplified version of the potential defined in (4), utilized in [16], is $V_{c}^{s 1}(d(\mathbf{p}))=\mathrm{k} \frac{1}{d(\mathbf{p})}$, with the respective control signal being active in the entire state space since $\frac{\partial V_{c}^{s}(d(\mathbf{p}))}{\partial \mathbf{p}} \rightarrow 0$ only when $d(\mathbf{p}) \rightarrow \infty$. Then, the minimum value set $\mathcal{S}$ is empty. It is utilized to shape an attractive potential to a goal $p_{T}$, as shown in Figure 2 but yields a combined potential that is not minimum at the target.

Other types of AP fields, utilized for joint limit avoidance in [17], combine logarithmic functions in a non-linear fashion:

$$
V_{c}(\mathbf{p})=\frac{\mathrm{k}_{1}}{2}\left(V_{L 1}-V_{H 1}\right)^{2}+\ldots+\frac{\mathrm{k}_{\mathrm{m}}}{2}\left(V_{L \mathrm{~m}}-V_{H \mathrm{~m}}\right)^{2},
$$

with $\mathrm{k}_{i} \in \mathbb{R}_{>}, i=1, \ldots, \mathrm{m}$ positive parameters, and

$$
V_{L i}\left(p_{i}\right)=\ln \underline{d}\left(p_{i}\right), V_{H i}\left(p_{i}\right)=\ln \bar{d}\left(p_{i}\right), i=1, \ldots, \mathrm{m},
$$

with $\mathbf{p}=\left[p_{1}, \ldots, p_{\mathrm{m}}\right]^{T}$ being, in this case, the joint positions of the robot, $m$ being the degrees of freedom and

$$
\underline{d}\left(p_{i}\right)=p_{i}-\underline{\mathrm{p}}_{i}, \bar{d}\left(p_{i}\right)=\overline{\mathrm{p}}_{i}-p_{i},
$$
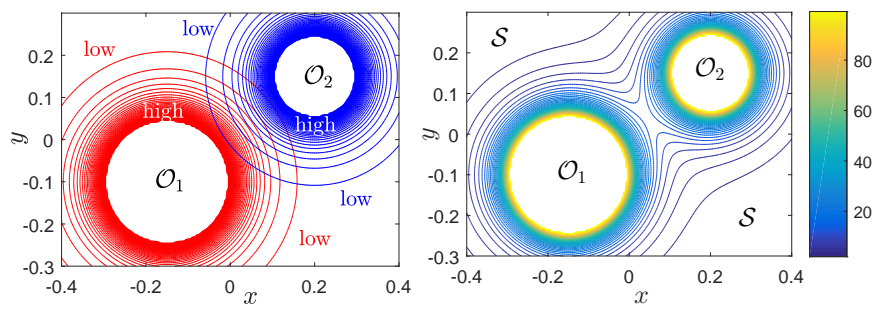

(a) [Spheres] Separate poten- (b) [Spheres] Combined potentials $V_{c 1}, V_{c 2}$

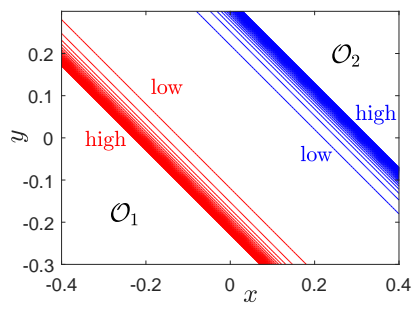
tial field $V_{c}$

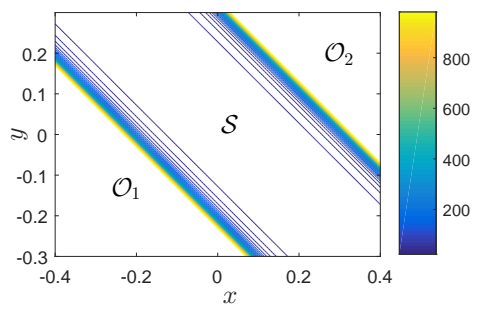

(c) [Planes] Separate potentials (d) [Planes] Combined potential $V_{c 1}, V_{c 2}$ field $V_{c}$

Fig. 1: Examples of AP fields.

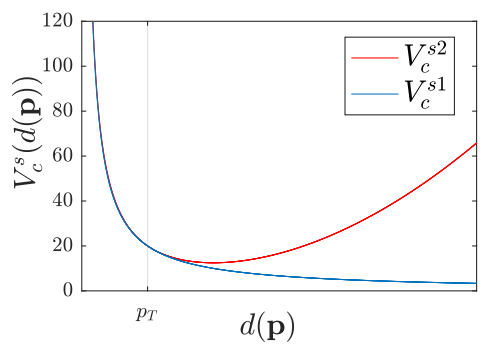

Fig. 2: Shaped attractive potential (red line) by the AP $\mathrm{k} d(\mathbf{p})^{-1}$ (blue line).

being the distance form the lower $\underline{p}_{i}$ and higher $\bar{p}_{i}$ joint limits respectively. By differentiating $V_{c}(\mathbf{p})$ w.r.t. $\mathbf{p}$, we get:

$$
\frac{\partial V_{c}(\mathbf{p})}{\partial p_{i}}=\mathrm{k}_{i}\left(V_{L i}-V_{H i}\right) \frac{\overline{\mathrm{p}}_{i}-\underline{\mathrm{p}}_{i}}{\left(p_{i}-\underline{\mathrm{p}}_{i}\right)\left(\overline{\mathrm{p}}_{i}-p_{i}\right)},
$$

which is used in (2). Figure 3 illustrates the AP field in this case. Notice that $\Omega$ forms an m-dimensional hyperrectangle in the joint space and $\frac{\partial V_{c}(\mathbf{p})}{\partial p_{i}}$ is zero only at $\mathbf{p}=\mathbf{p}_{e}=\frac{\overline{\mathbf{p}}+\underline{\mathbf{p}}}{2}$ which is the centroid of the hyperrectangle.

A similar approach considering ellipsoidal obstacle avoidance is presented in [18], for a robotic system driven to a desired target by a linear second order dynamical system. It is shown that when the obstacle avoidance signal acts alone, there is a compact set of local minima $\mathcal{S}$ in which $\frac{\partial V_{c}(\mathbf{p})}{\partial \mathbf{p}}=\mathbf{0}$. Figure 4 illustrates the AP field in case of two obstacles in a $2-\mathrm{D}$ world. Notice that $\mathcal{S}$ forms a $1-\mathrm{D}$ curve between the boundaries of $\mathcal{O}_{1}$ and $\mathcal{O}_{2}$.

\section{MOTIVATION}

Consider a N-dof non-redundant manipulator working on an N-dimensional operational space (joint or end-effector 


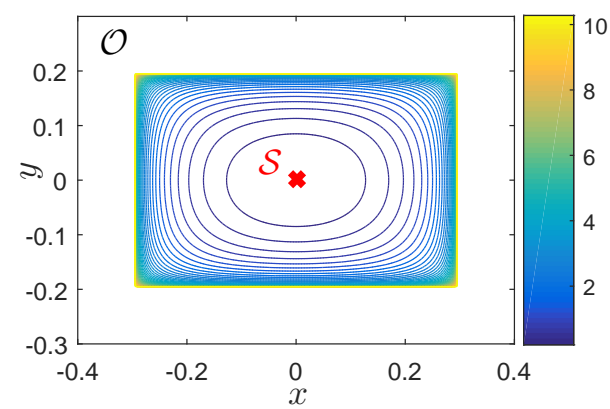

Fig. 3: Joint limit avoidance AP field.

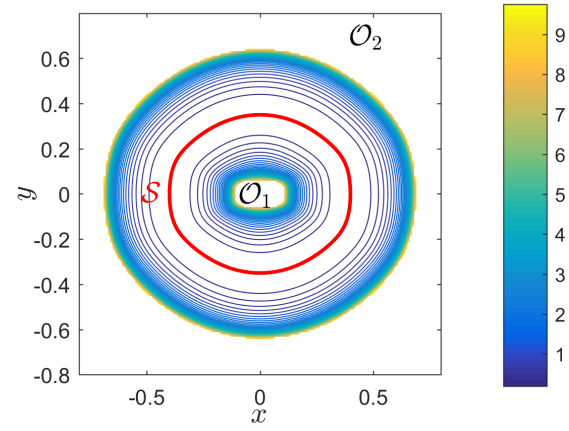

Fig. 4: Obstacle avoidance AP field, based on PPC.

space), in which the virtual constraints should be imposed, with gravity compensation, an additive dissipative force and virtual constraint control under the kinesthetic guidance of the human force. The dynamic model of the system is:

$$
\boldsymbol{\Lambda}_{\mathbf{p}}(\mathbf{p}) \ddot{\mathbf{p}}+\left(\mathbf{C}_{p}(\mathbf{p}, \dot{\mathbf{p}})+\mathbf{D}_{p}\right) \dot{\mathbf{p}}-\mathbf{u}_{c}=\mathbf{F}_{p},
$$

with $\mathbf{F}_{p} \in \mathbb{R}^{\mathrm{N}}$ being the generalized human force input to the system mapped appropriately in the operational space, $\mathbf{D}_{p} \in \mathbb{R}^{\mathrm{N} \times \mathrm{N}}$ a positive definite diagonal matrix representing a virtual dissipation, $\boldsymbol{\Lambda}_{p}(\mathbf{p}) \in \mathbb{R}^{\mathrm{N} \times \mathrm{N}}$ the inertia matrix, $\mathbf{C}_{p}(\mathbf{p}, \dot{\mathbf{p}}) \in \mathbb{R}^{\mathrm{N} \times \mathrm{N}}$ the Coriolis and centripetal matrix, and $\mathbf{u}_{c} \in \mathbb{R}^{N}$ is a constraint control signal (2) that is intended to confine the robot position in the constraint-free space $\Omega=$ $\mathbb{R}^{\mathrm{N}}-\mathcal{O}$ where $\mathcal{O} \subset \mathbb{R}^{\mathrm{N}}$. Notice that $\boldsymbol{\Lambda}_{p}$ is positive definite and the matrix $\dot{\boldsymbol{\Lambda}}_{p}-2 \mathbf{C}_{p}$ is skew symmetric, assuming a non-singular and one-to-one mapping between the velocities on the joint space and the operational space of interest.

Our aim, is to analyze the state boundedness of this system and consequently the constraint's satisfaction. Most of the robotic's literature only partially analyze the stability of this system; in pHRI related papers the stability analysis is confined to the proof of the system's passivity with respect to the system velocity output, while in the regulation problem which includes an attractive potential to the goal $\mathbf{p}_{T}$ [11], the stability analysis is confined to the goal's global asymptotic stability without considering the effect of inputs like the external force $\mathbf{F}_{p}$. The latter appears in many cases including noisy state measurements, unintentional contacts and kinesthetic guidance of a robot under impedance control without inertia shaping with known task related equilibrium. In general, as shown in [14] the system fails to satisfy the ISS criteria. Motivated by [14], we have simulated a RevolutePrismatic (RP) manipulator with joint variables $\mathbf{p}=\left[\begin{array}{ll}\theta & r\end{array}\right]^{T}$ with the joint space being the operational space (Fig. 5). Its inertia and Coriolis matrix is given below with $\mathrm{M}$ denoting the first link's mass at a distance $\mathrm{L}$ and $\mathrm{m}$ the mass of second link.

$$
\boldsymbol{\Lambda}_{p}(\mathbf{p})=\left[\begin{array}{cc}
\mathrm{m} r^{2}+\frac{\mathrm{ML}^{2}}{3} & 0 \\
0 & \mathrm{~m}
\end{array}\right], \mathbf{C}_{p}(\mathbf{p}, \dot{\mathbf{p}})=\mathrm{m} r\left[\begin{array}{cc}
\dot{r} & \dot{\theta} \\
-\dot{\theta} & 0
\end{array}\right]
$$

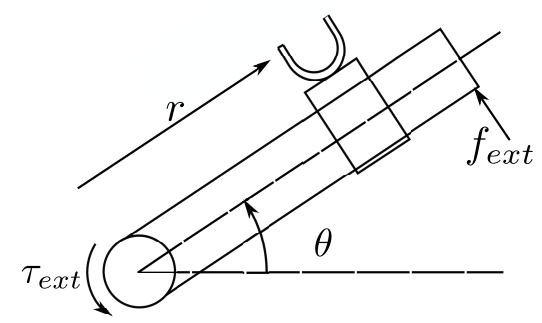

Fig. 5: Revolute-Prismatic (RP) manipulator

We impose joint limit virtual constraints via four potentials of the type (4) with respective gains $\mathrm{k}_{\theta}=100, \mathrm{k}_{r}=100$ and influence distance $\mathrm{d}_{0}=\overline{\mathrm{d}_{0}}=2.09 \times 10^{-2}(\mathrm{rad}$ or $\mathrm{m})$ to impose bounds $\underline{\mathrm{r}}=\overline{0 \mathrm{~m}}, \overline{\mathrm{r}}=1 \mathrm{~m}, \underline{\theta}=-\frac{\pi}{3} \mathrm{rad}, \bar{\theta}=\frac{\pi}{3}$ rad, in the joints $q \in\{\theta, r\}$, thus constrainig bilaterally each joint variable as depicted in Fig. 6. Hence, the constraint control signal (5) is applied. We consider an external force $\mathbf{f}_{\text {ext }}$ applied by the user in the end of the first link (Fig. 5), which yields only a torque in the first joint, hence $\mathbf{F}_{p}=$ $\left[\tau_{\text {ext }} 0\right]^{T}$, with $\tau_{\text {ext }}$ shown in Fig. 7 given by:

$$
\tau_{\text {ext }}= \begin{cases}3 & \text { if } \tau_{1}>3 \\ \tau_{1} & \text { if }-3 \leq \tau_{1} \leq 3 \\ -3 & \text { if } \tau_{1}<-3\end{cases}
$$

where $\tau_{1}=10 \cos \left(2 \pi T\left(\gamma^{\frac{t}{T}}-1\right) \frac{f_{0}}{\ln (\gamma)}\right)$ with $T=100$ sec being the total duration of simulation, $\gamma=\frac{f_{T}}{f_{0}} \in \mathbb{R}^{+}$ with $f_{0}=1 \mathrm{~Hz}, f_{T}=0.2 \mathrm{~Hz}$ being the initial and final

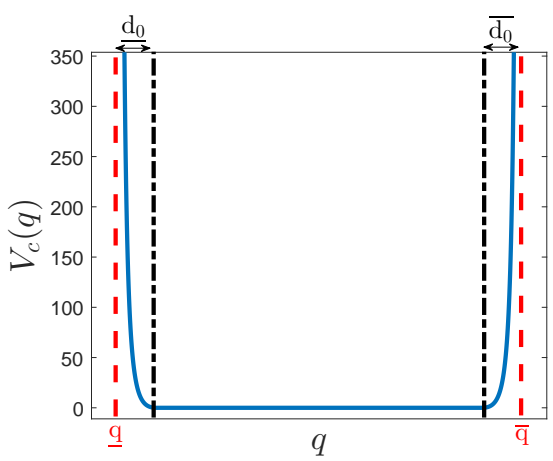

Fig. 6: The artificial potential with bilateral joint constraints. 


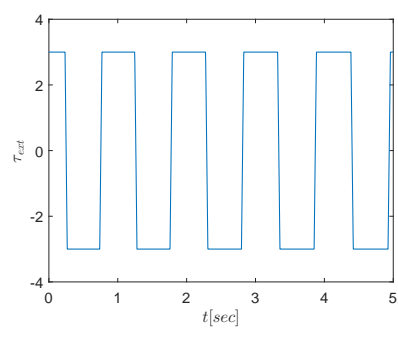

(a)

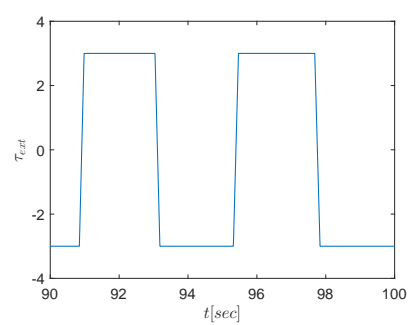

(b)
Fig. 7: Input signal. (a) First 5 seconds, (b) last 10 seconds.

frequencies respectively. We have simulated the system in MATLAB utilizing ode $15 \mathrm{~s}$ with values $\mathrm{ML}^{2}=0.1 \mathrm{Kg} \times \mathrm{m}^{2}$, $m=1.5 \mathrm{Kg}, \mathbf{D}_{p}=\operatorname{diag}\left(\left[\begin{array}{ll}2 & 1\end{array}\right]\right)$ from initial state $\mathbf{p}(0)=$ $\left[\begin{array}{lll}0 \mathrm{rad} & 0.3 \mathrm{~m}\end{array}\right]^{T}$ including the case of the prismatic joint being controlled to stay at its initial position via the control signal $-0.95(r-r(0))$. Figure 8 shows the response of joint positions without (Fig. 8.a) and with (Fig. 8.b) position control in $r$. Notice the nonlinear resonance behavior in both cases that leads the prismatic joint $r$ to the constraint even when it is position controlled.
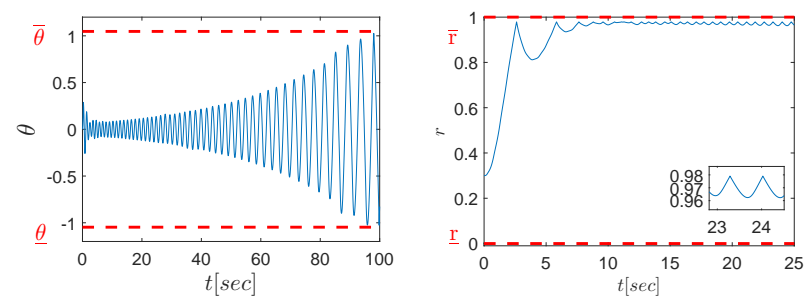

(a)
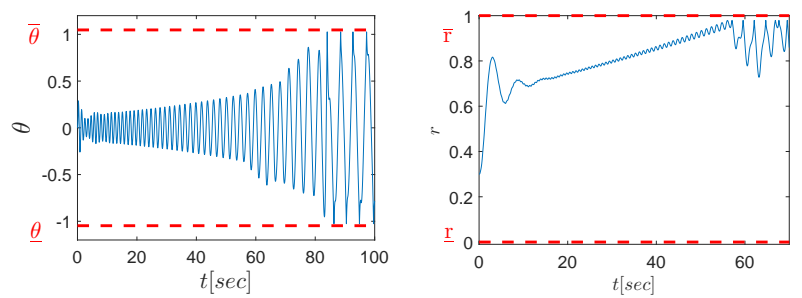

(b)

Fig. 8: Joint variable $\theta$ and $r$ response with $\tau_{\text {ext }}$ input (a) case of pure damping (b) case of a position controlled prismatic joint.

\section{STABILITY ANALYSIS}

In the stability analysis, we investigate the system's output passivity and zero-output dissipativity, the asymptotic stability of the unforced system's equilibrium set and the system's state boundedness and output passivity when the external input is of finite energy last concluding with a discussion on the consequences of our findings. To proceed with the stability analysis, we initially write system (10) in statespace. To this purpose we define $\xi=V_{c}(d(\mathbf{p}))$ as an internal system state and utilizing the state vector $\mathbf{s}=\left[\begin{array}{lll}\mathbf{p}^{T} & \dot{\mathbf{p}}^{T} & \xi\end{array}\right]^{T}$ we can write system (10) in state-space as follows:

$$
\dot{\mathbf{s}}=\mathbf{h}\left(\mathbf{s}, \mathbf{F}_{p}\right), \mathbf{s}_{0}=\mathbf{s}\left(t_{0}\right) \in D
$$

where

$$
\begin{gathered}
D=\left\{\mathbf{s}: \mathbf{s} \in \Omega \times \mathbb{R}^{\mathrm{N}} \times \mathbb{R}\right\} \\
\left.\mathbf{h}\left(\mathbf{s}, \mathbf{F}_{p}\right)=\left[\begin{array}{c}
\dot{\mathbf{p}} \\
\boldsymbol{\Lambda}_{p}^{-1}\left(-\left(\mathbf{C}_{p}+\underset{\left.\mathbf{D}_{p}\right) \dot{\mathbf{p}}}{-\mathbf{u}_{c} \dot{\mathbf{p}}}\right.\right.
\end{array} \mathbf{u}_{c}+\mathbf{F}_{p}\right)\right] .
\end{gathered}
$$

Theorem 1. Under the exertion of an external generalized human force $\mathbf{F}_{p}$, the system (12) is

1) strictly output passive,

2) zero-output dissipative

with respect to the output $\dot{\mathbf{p}}, \forall t \in\left[t_{0}, \tau\right]$ where $\tau \in\left(t_{0}, \infty\right)$.

Proof. Function $\mathbf{h}\left(\mathbf{s}, \mathbf{F}_{p}\right)$ is continuous in $t$ and locally Lipschitz with respect to $\mathbf{s}$. Owing to Theorem 3.1 in [19], there exists some $\tau>t_{0}$ such that the state equation $\dot{\mathbf{s}}=\mathbf{h}\left(\mathbf{s}, \mathbf{F}_{p}\right)$ with $\mathbf{s}\left(t_{0}\right)=\mathbf{s}_{0}$ has a unique solution in a maximal time interval $\left[t_{0}, \tau\right]$ with $\tau \in\left(t_{0}, \infty\right)$.

We assume the following candidate Lyapunov-like function:

$$
V=\frac{1}{2} \dot{\mathbf{p}}^{T} \boldsymbol{\Lambda}_{p} \dot{\mathbf{p}}+V_{c}(d(\mathbf{p})) \geq 0 .
$$

Taking the time derivative $\forall t \in\left[t_{0}, \tau\right]$ of (13) yields

$$
\dot{V}=\frac{1}{2} \dot{\mathbf{p}}^{T} \dot{\boldsymbol{\Lambda}}_{p} \dot{\mathbf{p}}+\dot{\mathbf{p}}^{T} \boldsymbol{\Lambda}_{p} \ddot{\mathbf{p}}+\frac{\partial V_{c}(d(\mathbf{p}))}{\partial \mathbf{p}} \dot{\mathbf{p}} .
$$

Substituting $\boldsymbol{\Lambda}_{p} \ddot{\mathbf{p}}$ from (10) in (14), and utilizing the skewsymmetry of matrix $\left(\dot{\boldsymbol{\Lambda}}_{p}-2 \mathbf{C}_{p}\right)$, yields:

$$
\dot{V}=-\dot{\mathbf{p}}^{T} \mathbf{D}_{p} \dot{\mathbf{p}}+\mathbf{F}_{p}^{T} \dot{\mathbf{p}} \leq \mathbf{F}_{p}^{T} \dot{\mathbf{p}}, \quad \forall t \in\left[t_{0}, \tau\right] .
$$

Hence, system (12) is strictly output passive for $t \in\left[t_{0}, \tau\right]$. Rewriting (15) by completing the squares, yields:

$$
\begin{aligned}
\dot{V} & \left.=-\| \sqrt{\mathbf{D}_{p}} \dot{\mathbf{p}}-\frac{1}{2} \sqrt{\mathbf{D}_{p}^{-1}} \mathbf{F}_{p}\right) \|^{2}+\frac{1}{4} \mathbf{F}_{p}^{T} \mathbf{D}_{p}^{-1} \mathbf{F}_{p} \\
& \leq \frac{1}{4} \mathbf{F}_{p}^{T} \mathbf{D}_{p}^{-1} \mathbf{F}_{p}, \forall t \in\left[t_{0}, \tau\right] .
\end{aligned}
$$

Notice that $\mathbf{F}_{p}$ is caused by the force applied by the human to the robot. Thus, $\mathbf{F}_{p}$ and $\mathbf{F}_{p}^{T} \mathbf{D}_{p}^{-1} \mathbf{F}_{p}$ are bounded functions of time. Hence, we can find a $\mathcal{K}$-function $\gamma$ such that

$$
\dot{V}<\gamma\left(\left|\mathbf{F}_{p}\right|\right), \forall t \in\left[t_{0}, \tau\right] .
$$

Therefore according to Definitions 2 and 3 in the Appendix, the system is zero-output dissipative for all $t \in\left[t_{0}, \tau\right]$.

Theorem 2. For the unforced system (12):

$$
\dot{\mathbf{s}}=\mathbf{h}\left(\mathbf{s}, \mathbf{0}_{\mathrm{N} \times 1}\right), \mathbf{s}_{0}=\mathbf{s}\left(t_{0}\right) \in D
$$

1) There is a compact set $D_{1} \subset D$ which is positively invariant with respect to (18).

2) the equilibrium $\mathbf{s}_{e}=\left[\begin{array}{lll}\mathbf{p}_{e}^{T} & \mathbf{0}_{1 \times \mathrm{N}} & 0\end{array}\right]^{T}$ where $\mathbf{p}_{e} \in \mathcal{S}$ is globally asymptotically stable.

Proof. Function $\mathbf{h}\left(\mathbf{s}, \mathbf{0}_{\mathrm{N} \times 1}\right)$ is continuous in $t$ and locally Lipschitz with respect to $\mathbf{s}$. Owing to Theorem 3.1 in [19], there exists some $\tau_{f}>t_{0}$ such that the state equation 
$\dot{\mathbf{s}}=\mathbf{h}\left(\mathbf{s}, \mathbf{0}_{\mathrm{N} \times 1}\right)$ with $\mathbf{s}\left(t_{0}\right)=\mathbf{s}_{0}$ has a unique solution in a maximal time interval $\left[t_{0}, \tau_{f}\right]$ with $\tau_{f} \in\left(t_{0}, \infty\right)$.

Consider the following candidate Lyapunov-like function:

$$
V=\frac{1}{2} \dot{\mathbf{p}}^{T} \boldsymbol{\Lambda}_{p} \dot{\mathbf{p}}+\frac{1}{2} V_{c}(d(\mathbf{p}))+\frac{1}{2} \xi \geq 0 .
$$

Taking the time derivative of (19) yields

$$
\dot{V}=\frac{1}{2} \dot{\mathbf{p}}^{T} \dot{\boldsymbol{\Lambda}}_{p} \dot{\mathbf{p}}+\dot{\mathbf{p}}^{T} \boldsymbol{\Lambda}_{p} \ddot{\mathbf{p}}+\frac{\partial V_{c}(d(\mathbf{p}))}{\partial \mathbf{p}} \dot{\mathbf{p}} .
$$

Substituting $\boldsymbol{\Lambda}_{p} \ddot{\mathbf{p}}$ from (10) in (20), and utilizing the skewsymmetry of matrix $\left(\dot{\boldsymbol{\Lambda}}_{p}-2 \mathbf{C}_{p}\right)$, yields:

$$
\dot{V}=-\dot{\mathbf{p}}^{T} \mathbf{D}_{p} \dot{\mathbf{p}} \leq 0, \forall t \in\left[t_{0}, \tau_{f}\right] .
$$

As $\dot{V}$ is negative semidefinite and $V$ is lower bounded by zero we can conclude the boundedness of $\dot{\mathbf{p}}(t)$ and $\xi(t)$ for all $t \in\left[t_{0}, \tau_{f}\right]$. Thus, there exist compact sets $\Omega_{\xi}, \Omega_{v}$ such that $\xi \in \Omega_{\xi} \subset \mathbb{R}$ and $\dot{\mathbf{p}} \in \Omega_{v} \subset \mathbb{R}^{\mathrm{N}}$ for all $t \in\left[t_{0}, \tau_{f}\right]$. Furthermore, $\xi \leq \bar{\epsilon}_{i}$ for some positive constant $\bar{\epsilon}_{i}$, which implies a bounded $\mathbf{p}$ along the gradient direction, hence $\mathbf{p}$ will not reach the boundary of the constrained region. When $\mathbf{p} \in \mathcal{S}$, i.e. $\mathbf{u}_{c}=\mathbf{0}$, as well as in the orthogonal space of $\mathbf{u}_{c}$ it is possible to prove using the system's kinetic energy that the velocity norm $\|\dot{\mathbf{p}}\|$ will exponentially converge to zero which implies that $\mathbf{p}$ will converge to $\mathbf{p}_{e} \in \mathcal{S}$, according to Lemma 2 (Appendix). Therefore the system (18) is 0-GAS for all $t \in\left[t_{0}, \tau_{f}\right]$ and $\mathbf{p}$ evolves in $\Omega_{1}$ a compact subset of $\Omega$. Consequently, $\mathbf{s}(t) \in D_{1} \triangleq \Omega_{1} \times \Omega_{v} \times \Omega_{\xi}$, a compact subset of $D$ for all $t \in\left[t_{0}, \tau_{f}\right]$. Using Theorem 3.3 in [19] we can conclude that $\tau_{f}$ can be extended to $\infty$ and the solution $\mathbf{s}(t)$ of (18) lies entirely in $D_{1}$, thus completing the proof.

Remark 2. The above result is similar to that obtained in region reaching control with motion constraints examined in [12]

Theorem 3. Consider the system (12) under the exertion of a generalized human force with finite energy $\mathbf{F}_{p} \in \mathcal{L}_{2}$. The following statement is valid: The system (12) is integral input to state stable (iISS).

Proof. Invoking Theorem 4 of the Appendix, given Theorem 1 and 2 we conclude that the system (12) is iISS $\forall t \in\left[t_{0}, \tau\right]$. Hence, there exists a function $\rho \in \mathcal{K} \mathcal{L}$ and $\rho_{1}, \rho_{2} \in \mathcal{K}_{\infty}$ such that for all $t \in\left[t_{0}, \tau\right]$ :

$$
\left|\mathbf{s}\left(t, \mathbf{s}_{0}, \mathbf{u}\right)\right| \leq \rho\left(\left|\mathbf{s}_{0}\right|, t\right)+\rho_{1}\left(\int_{0}^{t} \rho_{2}\left(\left|\mathbf{F}_{p}(\tau)\right|\right) d \tau\right) .
$$

Thus, $\mathbf{s}(t)$ is bounded under the exertion of finite energy human force for all $t \in\left[\begin{array}{ll}t_{0} & \tau\end{array}\right]$ or otherwise there exist a compact set $D^{\prime} \subset D$ in which every solution $\mathbf{s}(t)$ of (12) lies for all $t \in\left[t_{0}, \tau\right]$. Using Theorem 3.3 [19] we can conclude that $\tau$ can be extended to $\infty$ thus concluding the proof.

Lemma 1. Consider system (12) under the exertion of a generalized human force with finite energy $\mathbf{F}_{p} \in \mathcal{L}_{2}$. Then (12) is

1) strictly output passive,

\section{2) zero-output dissipative}

with respect to the output $\dot{\mathbf{p}}, \forall t \in\left[t_{0}, \infty\right)$.

Proof. This is an immediate consequence of Theorem 3 and Theorem 1.

To demonstrate the results we have simulated the RPmanipulator for both cases under the exertion of a truncated external input $\tau_{\text {ext }}$ at time $t_{r}=72$ as shown in Fig. 9. The joint position response shown in Fig. 10 is clearly bounded. Notice the different time scales utilized for clarity. Nevertheless, it is desirable in practice to avoid driving the unaffected link to its limit or induce oscillatory responses as shown in the response of $r$ in the first and second case. To this aim one should be able to characterize and avoid the inputs that would invoke such responses.

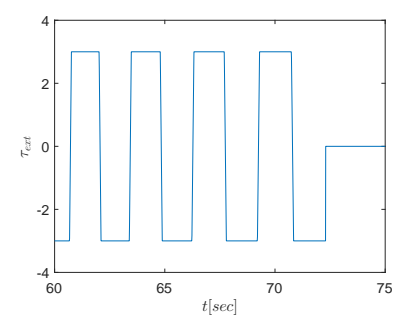

Fig. 9: Input with finite energy.
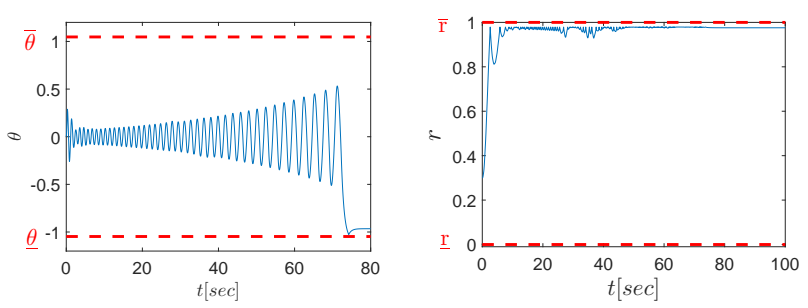

(a)
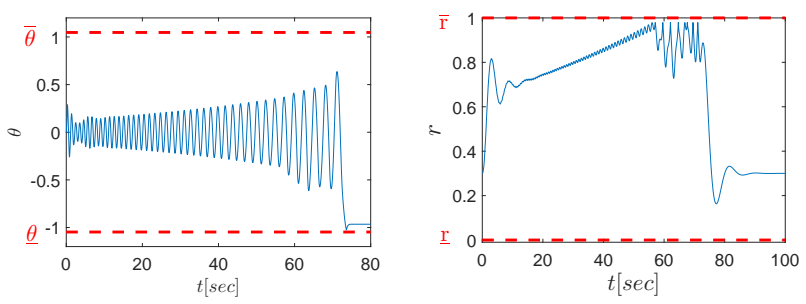

(b)

Fig. 10: Joint variable $\theta$ and $r$ response with finite energy input (a) case of pure damping (b) case of a position controlled prismatic joint

\section{CONCLUSIONS}

In this work we analyzed the output passivity and state boundedness of a robot under active constraints driven by the human force. It is proved that forces of finite energy guarantee state boundedness and hence constraint satisfaction. However, the response quality depends on the excitation of nonlinear resonance phenomena that occur in the presence of certain inputs and are undesirable in cases of pHRI. 


\section{APPENDIX}

\section{A. Convergence property}

Lemma 2. Suppose $\mathbf{f}:[0, \infty) \rightarrow \mathbb{R}^{w}$ and satisfies $\leq$ $|\mathbf{f}(t)| \leq n e^{-\lambda t} \forall t \in[0, \infty)$ where $n, \lambda>0$. Then $\int_{0}^{\infty} \mathbf{f}(t) d t$ converges.

Proof. Since we have $0 \leq|\mathbf{f}(t)| \leq n e^{-\lambda t} \forall t \in[0, \infty)$ and $\int_{0}^{\infty} n e^{-\lambda t} d t$ converges, by the comparison principle (see definition 1) we obtain that $\int_{0}^{\infty}|\mathbf{f}(t)| d t$ is convergent, thus using the fact that absolute convergence of the improper integral implies convergence, we have that $\int_{0}^{\infty} \mathbf{f}(t) d t$ converges.

Definition 1. Comparison Principle

If $0 \leq z(x) \leq b(x)$ for all $x \in[0, \infty)$ and $\int_{0}^{\infty} b(x) d x$ converges then $\int_{0}^{\infty} z(x) d x$ converges.

\section{B. Preliminaries about integral Input-to-State Stability}

Consider the system

$$
\begin{gathered}
\dot{\varphi}=\mathbf{W}(\boldsymbol{\varphi}, \mathbf{u}), \varphi_{0}=\varphi\left(t_{0}\right) \\
\mathbf{y}=\mathbf{H}(\varphi)
\end{gathered}
$$

where $\varphi \in \mathbb{R}^{\mathrm{n}}$ is the state, $\mathbf{y} \in \mathbb{R}^{\mathrm{p}}$ is the output and $\mathbf{u} \in \mathbb{R}^{\mathrm{m}}$ is the input of system (23). Let us further assume that the map $\mathbf{W}: \mathbb{R}^{\mathrm{n}} \times \mathbb{R}^{\mathrm{m}} \rightarrow \mathbb{R}^{\mathrm{n}}$ is locally Lipschitz, and the output is a continuous map $\mathbf{H}: \mathbb{R}^{\mathrm{n}} \rightarrow \mathbb{R}^{\mathrm{p}}$ with $\mathbf{H}\left(\mathbf{0}_{\mathrm{n} \times 1}\right)=\mathbf{0}_{\mathrm{p} \times 1}$.

Definition 2. ([14]) The system (23) is said to be smoothly dissipative if there exists a continuously differentiable, smooth and positive semidefinite function $V(\varphi)$ (called the storage function) together with a $\gamma_{2} \in \mathcal{K}$ and a continuous positive definite function $a_{1}$ such that

$$
\nabla V \mathbf{f}(\varphi, \mathbf{u}) \leq-a_{1}(|\mathbf{y}|)+\gamma_{2}(|\mathbf{u}|)
$$

for all $\varphi \in \mathbb{R}^{\mathrm{n}}$ and all $\mathbf{u} \in \mathbb{R}^{\mathrm{m}}$.

Definition 3. ([14]) The system (23) is zero-output (smoothly) dissipative, if (24) holds with $\mathbf{y}=\mathbf{0}_{\mathrm{p} \times 1}$, i.e., if there exists a (smooth) proper and positive definite $V(\varphi)$, and an $\gamma_{3} \in \mathcal{K}$, so that

$$
\nabla V \mathbf{f}\left(\mathbf{s}_{m}, \mathbf{u}_{m}\right) \leq \gamma_{3}(|\mathbf{u}|)
$$

for all $\varphi \in \mathbb{R}^{\mathrm{n}}$ and all $\mathbf{u} \in \mathbb{R}^{\mathrm{m}}$.

Definition 4. ([14]) The system (23) is said to be 0-GAS if the 0-input system:

$$
\dot{\varphi}=\mathbf{W}(\varphi, 0)
$$

is globally asymptotically stable (0-GAS).

Theorem 4. ([14]) When the system (23) is zero-output (smoothly) dissipative and 0-GAS then the system is iISS (integral Input-to-State Stable)

Theorem 5. ([20]) If the system (23) is iISS there exist function $\beta_{2} \in \mathcal{K} \mathcal{L}$ and $\mu_{1}, \mu_{2} \in \mathcal{K}_{\infty}$ such that

$$
\left|\mathbf{s}\left(t, \varphi_{0}, \mathbf{u}\right)\right| \leq \beta_{2}\left(\left|\varphi_{0}\right|, t\right)+\mu_{1}\left(\int_{0}^{t} \mu_{2}(|\mathbf{u}(\tau)|) d \tau\right) .
$$

\section{REFERENCES}

[1] L. B. Rosenberg, "The use of virtual fixtures as perceptual overlays to enhance operator performance in remote environments." DTIC Document, Tech. Rep., 1992.

[2] — - "Virtual fixtures: Perceptual tools for telerobotic manipulation," in Virtual Reality Annual International Symposium, 1993., 1993 IEEE. IEEE, 1993, pp. 76-82.

[3] Y. S. Park, X. Zhao, and S. Korthals, "Simulation of augmented telerobotic operation," in 2014 International Symposium on Optomechatronic Technologies, Nov 2014, pp. 242-246.

[4] S. A. Bowyer, B. L. Davies, and F. R. y Baena, "Active constraints/virtual fixtures: A survey," IEEE Transactions on Robotics, vol. 30, no. 1, pp. 138-157, Feb 2014

[5] S. A. Bowyer and F. R. y Baena, "Dissipative control for physical human-robot interaction," IEEE Transactions on Robotics, vol. 31, no. 6, pp. 1281-1293, 2015.

[6] A. Bettini, S. Lang, A. Okamura, and G. Hager, "Vision assisted control for manipulation using virtual fixtures: Experiments at macro and micro scales," in Robotics and Automation, 2002. Proceedings. ICRA'02. IEEE International Conference on, vol. 4. IEEE, 2002, pp. 3354-3361.

[7] A. Bettini, P. Marayong, S. Lang, A. M. Okamura, and G. D. Hager, "Vision-assisted control for manipulation using virtual fixtures," IEEE Transactions on Robotics, vol. 20, no. 6, pp. 953-966, 2004.

[8] J. E. Colgate, M. Peshkin, and S. H. Klostermeyer, "Intelligent assist devices in industrial applications: a review," in Proceedings 2003 IEEE/RSJ International Conference on Intelligent Robots and Systems (IROS 2003) (Cat. No.03CH37453), vol. 3, Oct 2003, pp. 2516-2521 vol.3.

[9] H. C. Lin, K. Mills, P. Kazanzides, G. D. Hager, P. Marayong, A. M. Okamura, and R. Karam, "Portability and applicability of virtual fixtures across medical and manufacturing tasks," in Proceedings 2006 IEEE International Conference on Robotics and Automation, 2006. ICRA 2006., May 2006, pp. 225-230.

[10] F. Rydn, A. Stewart, and H. J. Chizeck, "Advanced telerobotic underwater manipulation using virtual fixtures and haptic rendering," in 2013 OCEANS - San Diego, Sept 2013, pp. 1-8.

[11] O. Khatib and J. Le Maitre, "Dynamic control of manipulators operating in a complex environment," in On Theory and Practice of Robots and Manipulators, 3rd CISM-IFToMM Symp, vol. 267, 1978.

[12] C.-C. Cheah, "Region reaching control of robots with motion constraints," in Control, Automation, Robotics and Vision, 2008. ICARCV 2008. 10th International Conference on. IEEE, 2008, pp. 1752-1757.

[13] P. D. Triantafyllou, G. A. Rovithakis, and Z. Doulgeri, "Constrained visual servoing under uncertain dynamics," International Journal of Control, vol. 0, no. 0, pp. 1-13, 2018. [Online]. Available: https://doi.org/10.1080/00207179.2018.1428362

[14] D. Angeli, E. D. Sontag, and Y. Wang, "A characterization of integral input-to-state stability," IEEE Transactions on Automatic Control, vol. 45, no. 6, pp. 1082-1097, 2000.

[15] O. Khatib, "Commande dynamique dans l'espace opérationnel des robots manipulateurs en présence d'obstacles," PhD dissertation, Ecole Nationale Superieure de l'Aeronautique et de l'Espace, 1980.

[16] K. H. Wu, C. H. Chen, and J. D. Lee, "Genetic-based adaptive fuzzy controller for robot path planning," pp. 1687-1692 vol.3, 1996.

[17] D. Papageorgiou, A. Atawnih, and Z. Doulgeri, "A passivity based control signal guaranteeing joint limit avoidance in redundant robots," in 2016 24th Mediterranean Conference on Control and Automation (MED), June 2016, pp. 569-574

[18] S. Stavridis, D. Papageorgiou, and Z. Doulgeri, "Dynamical system based robotic motion generation with obstacle avoidance," IEEE Robotics and Automation Letters, vol. 2, no. 2, pp. 712-718, April 2017.

[19] H. Khalil, Nonlinear Systems, 3rd ed. Prentice Hall, 2002.

[20] A. Chaillet, D. Angeli, and H. Ito, "Combining iiss and iss with respect to small inputs: the strong iiss property," IEEE Transactions on Automatic Control, vol. 59, no. 9, pp. 2518-2524, 2014. 